\title{
Radiation Doses of Cerebral Blood Volume Measurements Using C-Arm CT: A Phantom Study
}

\author{
W.F. Chu, C.J. Lin, W.S. Chen, S.C. Hung, C.F. Chiu, T.H. Wu, and W.Y. Guo
}

\begin{abstract}
BACKGROUND AND PURPOSE: Parenchymal blood volume measurement by C-arm CT facilitates in-room peritherapeutic perfusion evaluation. However, the radiation dose remains a major concern. This study aimed to compare the radiation dose of parenchymal blood volume measurement using C-arm CT with that of conventional CTP using multidetector CT.
\end{abstract}

MATERIALS AND METHODS: A biplane DSA equipped with C-arm CT and a Rando-Alderson phantom were used. Slab parenchymal blood volume (8-cm scanning range in a craniocaudal direction) and whole-brain parenchymal blood volume with identical scanning parameters, except for scanning ranges, were undertaken on DSA. Eighty thermoluminescent dosimeters were embedded into 22 organ sites of the phantom. We followed the guidelines of the International Commission on Radiation Protection number 103 to calculate the effective doses. For comparison, 8-cm CTP with the same phantom and thermoluminescent dosimeter distribution was performed on a multidetector CT. Two repeat dose experiments with the same scanning parameters and phantom and thermoluminescent dosimeter settings were conducted.

RESULTS: Brain-equivalent dose in slab parenchymal blood volume, whole-brain parenchymal blood volume, and CTP were $52.29 \pm 35.31$, $107.51 \pm 31.20$, and $163.55 \pm 89.45 \mathrm{mSv}$, respectively. Variations in the measurement of an equivalent dose for the lens were highest in slab parenchymal blood volume (64.5\%), followed by CTP (54.6\%) and whole-brain parenchymal blood volume (29.0\%). The effective doses of slab parenchymal blood volume, whole-brain parenchymal blood volume, and CTP were $0.87 \pm 0.55,3.91 \pm 0.78$, and $2.77 \pm 1.59 \mathrm{mSv}$, respectively.

CONCLUSIONS: The dose measurement conducted in the current study was reliable and reproducible. The effective dose of slab parenchymal blood volume is about one-third that of CTP. With the advantages of on-site and immediate imaging availability and saving procedural time and patient transportation, slab parenchymal blood volume measurement using C-arm CT can be recommended for clinical application.

ABBREVIATIONS: PBV = parenchymal blood volume; s-PBV = slab parenchymal blood volume; $w$-PBV = whole-brain parenchymal blood volume; TLD = thermoluminescent dosimeter

$\mathbf{C}^{1}$ $\mathrm{P}$ is an imaging technique commonly used in conjunction with CT angiography to evaluate the cerebral perfusion status of patients with cerebrovascular disorders. ${ }^{1,2}$ It serves as an excellent patient triage tool for strategy planning in patients with acute ischemic stroke by its quantitative delineation of the extent of

Received September 16, 2013; accepted after revision October 12

From the Department of Radiology (W.F.C., C.J.L., S.C.H., C.F.C., W.Y.G.), Taipei Veterans General Hospital, Taipei, Taiwan; and School of Medicine (W.F.C., C.J.L.,

S.C.H., C.F.C., W.Y.G.) and Department of Biomedical Imaging and Radiological Sciences (W.S.C., T.H.W.), National Yang-Ming University, Taipei, Taiwan.

W.Y. Guo and T.H. Wu contributed equally and are co-corresponding authors to this work.

Please address correspondence to Wan Yuo Guo, MD, PhD, Department of Radiology, Taipei Veterans General Hospital, 201 Shipai Rd, Sec 2, Taipei, Taiwan, 11217; e-mail: wyguo@vghtpe.gov.tw; or Tung Hsin Wu, PhD, Department of Biomedical Imaging and Radiological Sciences, National Yang Ming University, 155 Li-Nong St, Sec. 2, Taipei, Taiwan 112; e-mail: tung@ym.edu.tw

http://dx.doi.org/10.3174/ajnr.A3822 ischemic core and penumbra. ${ }^{1,3,4}$ It is also used to evaluate therapeutic effects and detect complications such as hyperperfusion syndrome or hemorrhagic transformation for subsequent management. $^{5-7}$ Nevertheless, CT cannot demonstrate intracranial territorial hemodynamics. It is not feasible either to serve as imaging guidance for neurovascular intervention or to provide immediate information without patient transportation to scanners. Recent advances in flat panel detector technology render a C-arm system capable of providing projection radiography, fluoroscopy, DSA, and CT-like images in 1 imaging suite. Due to the versatile applications, C-arm CT equipped with a flat detector has gained wide popularity in current imaging practice..$^{8-10}$

C-arm CT also allows functional imaging of parenchymal blood volume (PBV) with either intravenous or intra-arterial contrast injection. ${ }^{11,12}$ It is highly correlated with CBV from CTP and has shown promising initial results in treating acute middle cerebral artery occlusions. ${ }^{13}$ It can be used to access the extent of 
Table 1: Number and distributions of TLD-100Hs in the RandoAlderson phantom for the experiments

\begin{tabular}{clc}
\hline Site Number & \multicolumn{1}{c}{ Organ } & TLD No. \\
\hline 1 & Brain & 21 \\
2 & Salivary gland & 4 \\
3 & Thyroid & 4 \\
4 & Esophagus & 3 \\
5 & Lung & 3 \\
6 & Heart & 3 \\
7 & Liver & 3 \\
8 & Stomach & 3 \\
9 & Kidney & 2 \\
10 & Small intestine & 4 \\
11 & Colon & 2 \\
12 & Bladder & 2 \\
13 & Gonad (surface) & 4 \\
14 & Rib & 2 \\
15 & Thoracic spine & 3 \\
16 & Lumbar spine & 3 \\
17 & Pelvis & 2 \\
18 & Skin (eye) & 4 \\
19 & Skin (thorax) & 4 \\
20 & Skin (abdomen) & 4 \\
& Total & 80 \\
\hline
\end{tabular}

the ischemic core and detect hyperperfusion phenomena peritherapeutically in the angiography suite, facilitating endovascular therapy without the transfer of patients to the CT room. ${ }^{10-15}$ In a recent study, Royalty et $\mathrm{al}^{16}$ showed the feasibility of using C-arm CT to measure perfusion parameters in an animal model. Nevertheless, the combination of DSA and flat panel parenchymal blood volume raises concerns related to radiation exposure. ${ }^{17,18}$ Unintentional adverse effects due to overexposure to $\mathrm{x}$-rays in CT rooms or angiography suites should be avoided. ${ }^{17,19}$ Before the one-stop imaging from diagnosis to imaging-guided endovascular treatment and posttherapeutic evaluation is widely adopted in clinical service, the dose measurement of $\mathrm{C}$-arm $\mathrm{CT}$ in perfusion parameter evaluation should be investigated. We, therefore, present the current study.

\section{MATERIALS AND METHODS}

\section{Radiation Dose Measurement}

A radiation experiment by using an anthropomorphic phantom (Rando-Alderson phantom; Radiology Support Devices, Long Beach, California) was used for both PBV and CTP. The phantom consisted of 35 sections of $2.5-\mathrm{cm}$ thickness each. It simulated the body of a 170-cm tall, 70-kg man. The phantom was composed of a natural human skeleton embedded in a mass with the properties of human soft tissue (mass attenuation, $\rho=0.985 \mathrm{~kg} / \mathrm{dm}^{3}$, effective atomic number, $Z_{\text {eff }}=7.3$ ). The thorax of the phantom was made of foam $\left(\rho=0.32 \mathrm{~kg} / \mathrm{dm}^{3} ; Z_{\text {eff }}=7.3\right)$ to simulate human lung tissue. Eighty lithium fluoride thermoluminescent dosimeters (TLD-100H; Bicron-Harshaw, Solon, Ohio) divided across 22 organ sites were embedded in the phantom (Table 1). Those TLD- $100 \mathrm{Hs}$ with coefficients of variation $>3 \%$ were excluded. The TLDs were made of a tissue-equivalent material with high sensitivity and a linear response to radiation doses. Moreover, after an adequate annealing process, the TLDs could be recycled, which made them suitable for monitoring the delivery of radiation with PBV and CTP imaging techniques. We followed the guidelines of the International Commission on Radiation Protection number 103 to calculate the effective dose (ED) $)^{20}$ as follows:

$$
E D=\sum D_{T} \cdot w_{R} \cdot w_{T}
$$

where $D_{T}$ is the mean absorbed dose to the target organ, $W_{R}$ is the radiation-weighting factor, and $W_{T}$ is the tissue-weighting factor. Accordingly, the $W_{R}$ for x-ray was 1 . The absorbed dose obtained from TLD in each specific organ was multiplied by the $W_{R}$ to yield the equivalent doses in each individual organ.

The values of the entrance skin dose/dose-area product of Carm CT and the dose-length product of multidetector CT as shown by the consoles were recorded for comparison, while both scanners were regularly calibrated following standard clinical service requirements.

\section{CT Perfusion}

For comparison, CT scans on the same phantom and TLD distribution were obtained on a 256-section multidetector CT scanner (Brilliance iCT; Philips Healthcare, Best, the Netherlands). A clinical CTP scanning protocol was used in accordance with the American Association of Physicists in Medicine (AAPM). ${ }^{21}$ The acquisition parameters were 8 - $\mathrm{cm}$ scan coverage identical to slab PBV (s-PBV), 5-mm section thickness, $64 \times 1.25 \mathrm{~mm}$ collimation, $20 \times 20 \mathrm{~cm} \mathrm{FOV}, 80 \mathrm{kV}$ (peak), and $250-\mathrm{mA}$ tube current with a rotation time of 0.4 seconds, equivalent to effective 100 mAs. The scan interval was 1.5 seconds, with a 1-minute duration. No patient table movement was encountered throughout the CTP data acquisition.

\section{Parenchymal Blood Volume}

PBV dose measurement was conducted with a biplane flat panel detector-DSA suite (Axiom-Artis; Siemens, Erlangen, Germany). A routine clinical imaging protocol (one 8-second rotational mask run with 60 frames/second followed by another 8 -second rotational contrast-medium-filled run with the same frame rate) for cerebral PBV measurement (syngo DynaPBV Neuro; Siemens) was used. The scanning parameters were $73 \mathrm{kV}, 483-555$ $\mathrm{mA}$, rotation angle of $200^{\circ}, 0.5^{\circ}$ per frame, and a $616 \times 480 \mathrm{ma}-$ trix. Two FOVs, full-size $(30 \times 40 \mathrm{~cm})$ and slab $(8 \mathrm{~cm}$ at the isocenter in a craniocaudal direction $\times 40 \mathrm{~cm}$ in a right-left direction), were used. The PBV measurements obtained were respectively called whole-brain PBV (w-PBV) and slab PBV (ranging from the sella turcica to high convexity). A repeat dose measurement with same imaging protocol and scanning parameters was conducted.

\section{RESULTS}

The dose-area product and entrance skin dose were 2574.6 $\mu \mathrm{Gym}^{2} / 222 \mathrm{mGy}$ and $6850.4 \mu \mathrm{Gym}^{2} / 238 \mathrm{mGy}$ for s-PBV and w-PBV, respectively. As a comparison, the CTP dose-length product was $1177.3-1232.9 \mathrm{mGy} \cdot \mathrm{cm}$, equivalent to an effective dose of 2.47-2.59 mSv calculated by the American Association of Physicists in Medicine of $\mathrm{k}$ factor $\left(\mathrm{k}=0.0021 \mathrm{mSv} \cdot \mathrm{mGy}^{-1} \cdot \mathrm{cm}^{-1}\right)$. The equivalent doses of different tissue organs in s-PBV, $\mathrm{w}-\mathrm{PBV}$, and CTP measured from TLDs are listed in Table 2. Most TLDs were located in the head, followed by the salivary glands and thyroid glands in all 3 protocols. s-PBV demonstrated a $62.5 \%$ ab- 
Table 2: Radiation doses of individual organs/tissues in CTP, s-PBV, and $w$-PBV protocols measured from 2 repeated TLD exposure experiments ${ }^{\mathrm{a}}$

\begin{tabular}{lccc}
\hline \multicolumn{1}{c}{$\begin{array}{c}\text { Tissue or } \\
\text { Organ }\end{array}$} & CTP & s-PBV & w-PBV \\
\hline Brain & $163.55 \pm 89.45$ & $52.29 \pm 35.31$ & $107.51 \pm 34.20$ \\
Salivary gland & $13.94 \pm 1.35$ & $3.03 \pm 0.61$ & $137.75 \pm 16.77$ \\
Thyroid & $4.44 \pm 1.64$ & $1.20 \pm 0.28$ & $12.68 \pm 2.48$ \\
Thymus & $0.23 \pm 0.08$ & $0.10 \pm 0.07$ & $0.46 \pm 0.15$ \\
Esophagus & $1.18 \pm 0.36$ & $0.60 \pm 0.08$ & $2.61 \pm 0.36$ \\
Breast & $0.61 \pm 0.17$ & $0.19 \pm 0.07$ & $1.16 \pm 0.17$ \\
Lung & $0.50 \pm 0.12$ & $0.28 \pm 0.09$ & $1.19 \pm 0.20$ \\
Stomach & $0.06 \pm 0.04$ & $0.07 \pm 0.06$ & $0.16 \pm 0.08$ \\
Liver & $0.08 \pm 0.03$ & $0.05 \pm 0.03$ & $0.19 \pm 0.07$ \\
Kidney & $0.64 \pm 0.74$ & $0.05 \pm 0.02$ & $0.18 \pm 0.03$ \\
Small intestine & $0.03 \pm 0.05$ & $0.03 \pm 0.05$ & $0.04 \pm 0.05$ \\
Colon & $0.00 \pm 0.00$ & $0.01 \pm 0.00$ & $0.01 \pm 0.01$ \\
Bladder & $0.05 \pm 0.03$ & $0.05 \pm 0.03$ & $0.08 \pm 0.05$ \\
Gonads & $0.06 \pm 0.03$ & $0.03 \pm 0.03$ & $0.07 \pm 0.04$ \\
Bone marrow & $0.43 \pm 0.15$ & $0.32 \pm 0.13$ & $1.12 \pm 0.36$ \\
Skin & $0.45 \pm 0.29$ & $0.12 \pm 0.06$ & $0.65 \pm 0.11$ \\
Bone surface & $0.42 \pm 0.15$ & $0.32 \pm 0.15$ & $1.12 \pm 0.50$ \\
Effective dose ${ }^{b}$ & $2.77 \pm 1.59$ & $0.87 \pm 0.55$ & $3.91 \pm 0.78$ \\
\hline
\end{tabular}

a Unless otherwise specified, data are in millisieverts.

bThe sum of organ doses are weighted by the tissue-weighting factor recommended in the International Commission on Radiation Protection number 103. sorbed dose reduction compared with w-PBV across all organ/ tissue sites. In addition to head and neck regions, w-PBV showed a higher dose than s-PBV in the remaining organs.

Brain-equivalent doses in s-PBV, w-PBV, and CTP were $52.29 \pm 35.31,107.51 \pm 31.20$, and $163.55 \pm 89.45 \mathrm{mSv}$, respectively. The variation of doses in brain areas was the largest in s-PBV (67.5\%), followed by CTP (54.7\%) and then by w-PBV $(31.8 \%)$ (Fig 1). The equivalent dose of the lens was highest in CTP $(157 \pm 141 \mathrm{mSv})$, followed by w-PBV $(104 \pm 25 \mathrm{mSv})$ and then s-PBV $(37 \pm 39 \mathrm{mSv})$. Variation in the measurement of equivalent dose for the lens was larger in CTP (54.6\%) and s-PBV (64.5\%) and lower in w-PBV (29.0\%) (Fig 2). The equivalent dose of salivary glands was largest in w-PBV followed by CTP and s-PBV (Fig 3). For the remaining organs below the thyroid, there were no dramatic variations in individual scanning protocols.

The effective dose was lowest in s-PBV $(0.87 \pm 0.55 \mathrm{mSv})$, followed by CTP $(2.77 \pm 1.59 \mathrm{mSv})$ and $\mathrm{w}-\mathrm{PBV}(3.91 \pm 0.78$ $\mathrm{mSv}$ ), according to International Commission on Radiation Protection number 103.

\section{DISCUSSION}

The effective dose of CTP in the current study measured by TLD $(2.77 \pm 1.59 \mathrm{mSv})$ is close to that estimated from the dose-length product (2.47-2.59 mSv), calculated by the American Association of Physicists in Medicine of $\mathrm{k}$ factor, the
A

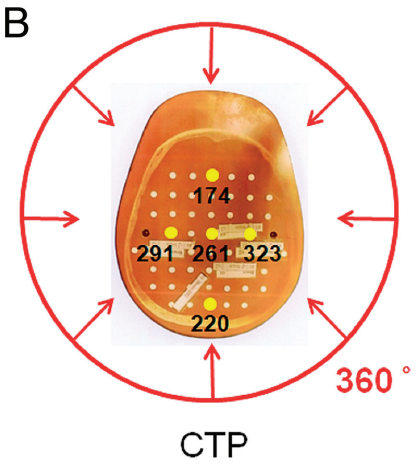

C

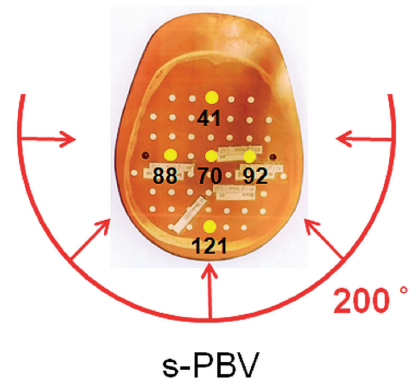

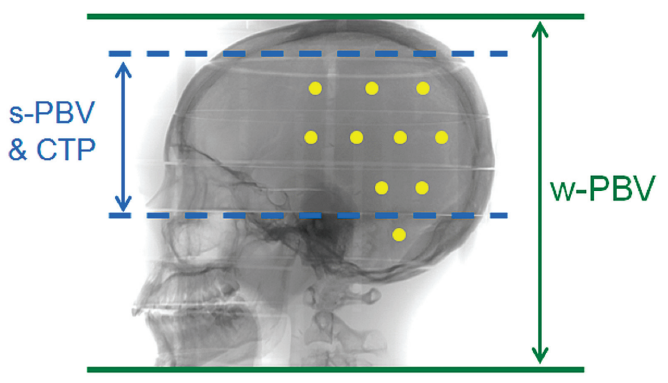

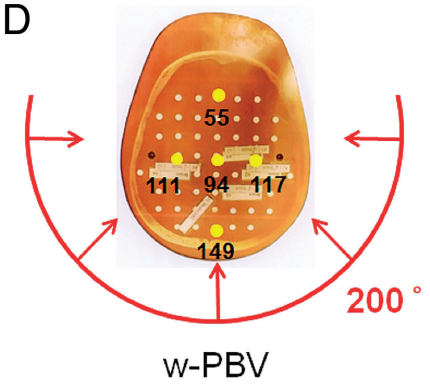

FIG 1. A, Ten yellow dots simulating TLDs are placed on a lateral skull view of the RandoAlderson phantom. The scanning ranges of s-PBV and CTP are both $8 \mathrm{~cm}$ at the isocenter in a craniocaudal direction (blue dashed lines), while that of $w-P B V$ is $30 \mathrm{~cm}$ (green solid lines). B, Eight-centimeter CTP is performed with a $360^{\circ}$ rotation of the $x$-ray source in CT, and both s-PBV and w-PBV ( $C$ and $D$ ) are obtained with a $200^{\circ}$ rotation of the $x$-ray source in DSA from the dorsal side of the Rando-Alderson phantom. The red arrows indicate $x$-ray beams. The 5 yellow dots placed on transaxial sections of the phantom indicate the TLDs placed on a phantom section. The numbers indicate the equivalent dose of each TLD in millisieverts. The elliptic head configuration of the phantom makes the dose heterogeneous (ie, the attenuation distance of TLDs on the ventral side of the phantom is longer than that on the dorsal side and the attenuation distance of the center one is longer than that of the peripheral one). The geometry makes the overall variation of the dose in the brain area the largest in s-PBV (67.5\%), followed by CTP (54.7\%) and then w-PBV (31.8\%). reference effective dose estimation in a clinical setting. Nevertheless, we found some heterogeneity of dose distribution in the current TLD dose readouts. The heterogeneity is caused by multifactorial mechanisms. The elliptic shape of the Rando-Alderson phantom and limited rotation angles of the tube $\left(200^{\circ}\right)$ make the attenuation distance of individual TLDs on the ventral side of the phantom longer than those on the dorsal side. Moreover, in the same section of the Rando-Alderson phantom, the attenuation distance of individual TLDs in the center is longer than that in the periphery. Therefore, dose values read in the ventral side and center are lower (Fig 1). This phenomenon is more profound in the area of the primary beam, such as in the brain and salivary glands. Narrower scan ranges (ie, s-PBV and CTP) cause higher dose variations than those in $\mathrm{w}-\mathrm{PBV}$. The latter received both primary and scatter beams of irradiation (Fig 3). Due to the vicinity of the inferior margin of the scanning range and lens in CTP and sPBV modes, the lenses were partly within the primary beams and resulted in a large variation of equivalent dose (Fig 2). In a clinical scenario, we can adjust the patient's head into the chin- 


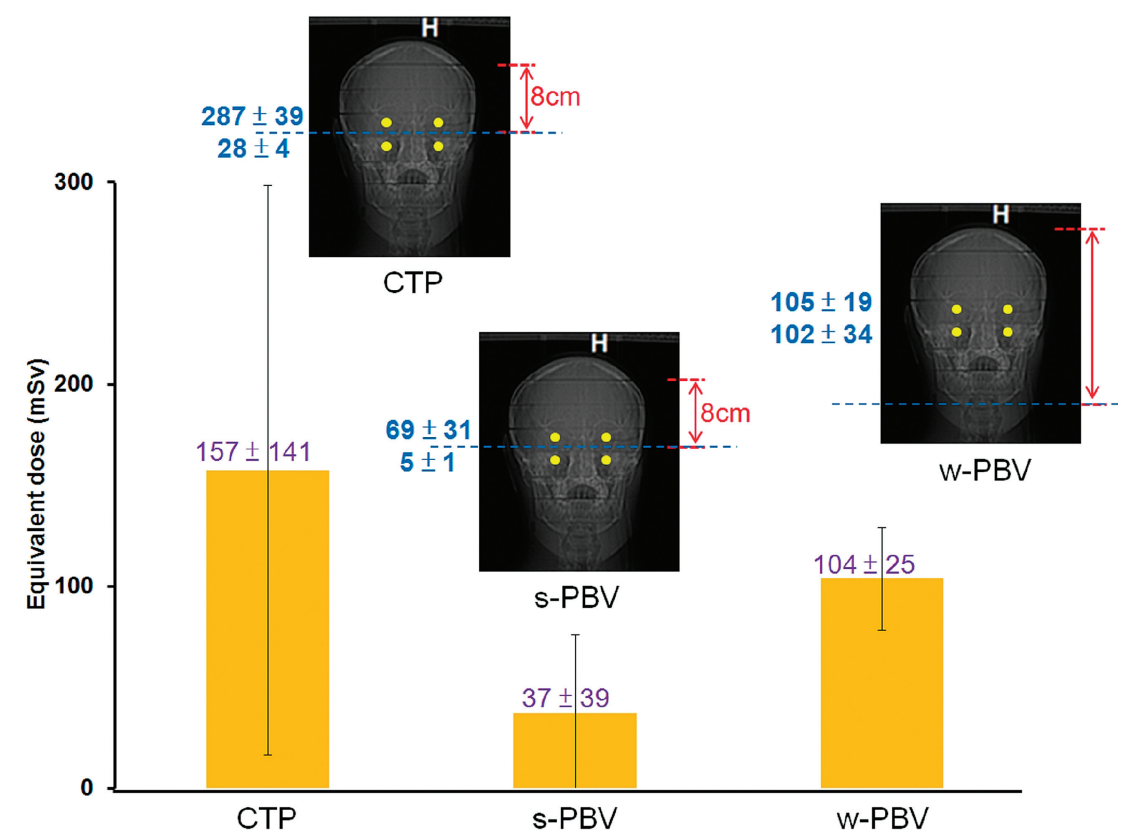

FIG 2. Equivalent dose (millisieverts) of the lens for CTP, s-PBV, and w-PBV. Variation in the measurement of the equivalent dose for the lens is larger in CTP $(54.6 \%)$ and s-PBV $(64.5 \%)$ and lower in w-PBV (29.0\%). Two TLDs are within the primary beams, and the other 2 TLDs are within the secondary beams in CTP and s-PBV, while in w-PBV, all TLDs are within the primary beam.

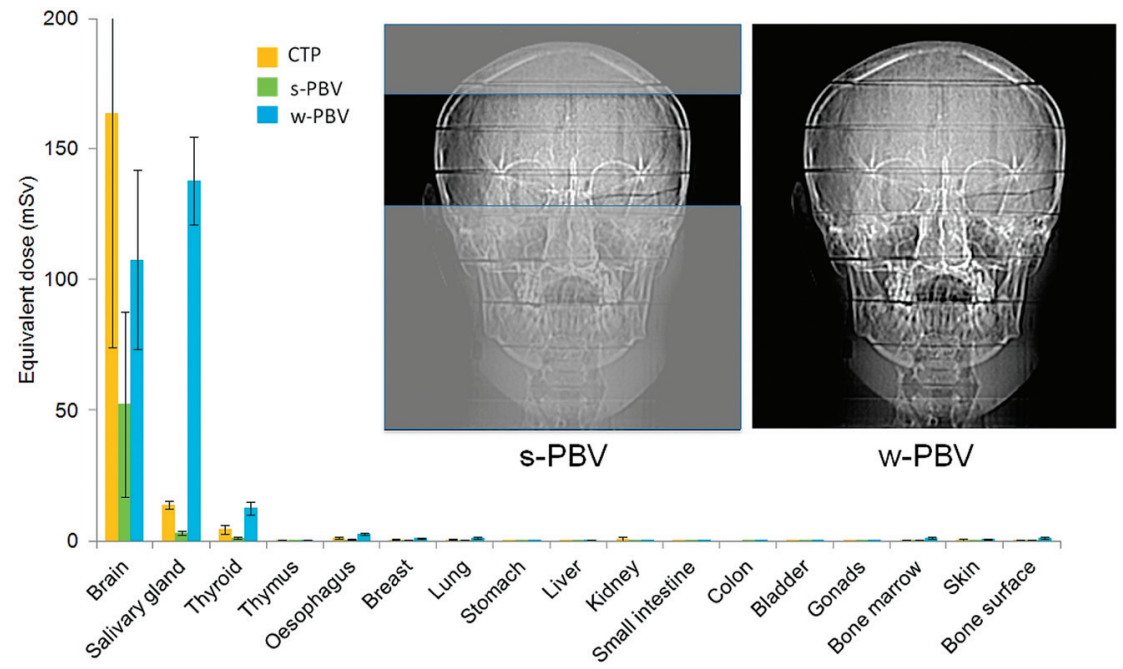

FIG 3. Anteroposterior views of the Rando-Alderson phantom for s-PBV and w-PBV. For the brain region, CTP received the largest equivalent dose followed by $w-P B V$ and s-PBV. For the salivary and thyroid gland regions, the equivalent dose of $w-P B V$ is higher than that of s-PBV and CTP due to larger $x$-ray beam z-axial coverage.

tuck position to avoid the lenses falling within the scan ranges in CTP or s-PBV.

CTP acquisition protocols were related to the scan interval, scan range, and tube potential. These variables were different across different brands and platforms of CT scanners. The effective doses, therefore, can range from 2.5 to $10 \mathrm{mSv} .^{22-25} \mathrm{We}$ chose the latest CT technique in our institution for the comparative study. The s-PBV (8-cm scanning range at the isocenter) was conducted for direct and equal comparison of radiation doses with CTP (8-cm coverage). Nevertheless, in contrast to the fixed and limited coverage of the brain for most CTPs, the scan range for
PBV can be adjusted according to the operator's preference. For example, coverage of the infratentorium is not mandatory for a patient with known acute MCA stroke, not only because of suboptimal quality infratentorially but also because of irrelevant vascular territory. ${ }^{26}$ In real practice, the coverage can be individually tailored to an optimal range on the basis of clinical needs. Although the s-PBV did not include the whole brain, it sufficiently covered most regions of interest, which are important for neurointerventional procedures. For example, vascular pathology that involves the distal anterior cerebral artery and MCA tributaries was usually too distal for endovascular intervention in acute stroke settings. ${ }^{26}$ Similarly, the imaging quality of infratentorium perfusion imaging was suboptimal due to beam-hardening artifacts; thus, its role in guiding thrombolytic therapy is limited. ${ }^{27}$

The comprehensive acute stroke imaging work-up usually includes noncontrast CT, CT angiography, and CT perfusion. ${ }^{28}$ Their main contributions are to exclude hemorrhage and large-territory infarction and to further quantify the thrombus burden and collateral circulations. $^{1,29,30}$ Modern flat panel detector-CT imaging, to some extent, has a spatial and contrast resolution equivalent to that of multidetector CT. ${ }^{14,31}$ For those patients eligible for intra-arterial thrombolysis, DSA is far superior to CT angiography for evaluating vasculatures, occluded length, and collaterals. In addition to PBV, in-room assessment of the ischemic core and thrombus condition was feasible, thus reducing the door-to-puncture time and improving recanalization rates. ${ }^{10,32}$

Further reduction of the radiation dose is possible by lowering tube currents in CTP. ${ }^{33} \mathrm{~A}$ similar strategy might be applied in PBV. The optimal tube voltage of the PBV system among individuals was beyond the scope of our study. The radiation dose from fluoroscopy required to put patients into an appropriate scan position before PBV differs between operators. Although its contribution to the overall dose is trivial, it can be further reduced with improving skill in scanning practice. Wearing lens protection while undergoing PBV may be an alternative to further reduce the dose to the lens.

s-PBV decreases the radiation dose and keeps an efficient coverage for peritherapeutic monitoring of cerebral hemody- 
namics. If combined with faster and multiple rotations, it is possible to obtain cerebral blood flow with reasonable radiation exposure. ${ }^{16}$ Because the arterial input function was determined by histogram rather than vessels, the measurements were relatively constant as long as most of the brain was included in the scanning range. ${ }^{11,12}$

\section{CONCLUSIONS}

The radiation dose measurement presented in this study is reliable and reproducible. Cerebral PBV measurement by using flat panel detector-DSA is a dose-saving imaging protocol. The effective dose with s-PBV is about one-third that of CTP. If one takes into account the advantages of on-site and immediate morphologic imaging availability and saving procedural time and patient transportation, flat panel DSA s-PBV may be recommended as an alternative peri-interventional imaging for evaluating intracranial hemodynamics of neurovascular disorders.

Disclosures: Wan You Guo-UNRELATED: Other: collaboration research contract between Taipei Veterans General Hospital and Siemens Healthcare. * Money paid to the institution.

\section{REFERENCES}

1. Konstas AA, Wintermark $M$, Lev MH. CT perfusion imaging in acute stroke. Neuroimaging Clin N Am 2011;21:215-38, ix

2. Waaijer A, van Leeuwen MS, van Osch MJ, et al. Changes in cerebral perfusion after revascularization of symptomatic carotid artery stenosis: CT measurement. Radiology 2007;245:541-48

3. Xue J, Gao P, Wang X, et al. Ischemic lesion typing on computed tomography perfusion and computed tomography angiography in hyperacute ischemic stroke: a preliminary study. Neurol Res 2008;30:337-40

4. Olivot JM, Mlynash M, Thijs VN, et al. Optimal Tmax threshold for predicting penumbral tissue in acute stroke. Stroke 2009;40:469-75

5. Fukuda T, Ogasawara K, Kobayashi M, et al. Prediction of cerebral hyperperfusion after carotid endarterectomy using cerebral blood volume measured by perfusion-weighted MR imaging compared with single-photon emission CT. AJNR Am J Neuroradiol 2007;28:737-42

6. Cheng B, Golsari A, Fiehler J, et al. Dynamics of regional distribution of ischemic lesions in middle cerebral artery trunk occlusion relates to collateral circulation. J Cereb Blood Flow Metab 2011;31:36-40

7. Molina CA, Montaner J, Abilleira S, et al. Timing of spontaneous recanalization and risk of hemorrhagic transformation in acute cardioembolic stroke. Stroke 2001;32:1079-84

8. Kyriakou Y, Struffert T, Dorfler A, et al. Basic principles of flat detector computed tomography (FD-CT) [in German]. Radiologe 2009;49:811-19

9. Jaffray DA, Siewerdsen JH. Cone-beam computed tomography with a flat-panel imager: initial performance characterization. Med Phys 2000;27:1311-23

10. Hung SC, Lin CJ, Guo WY, et al. Toward the era of a one-stop imaging service using an angiography suite for neurovascular disorders. Biomed Res Int 2013;2013:873614

11. Struffert T, Deuerling-Zheng Y, Engelhorn T, et al. Feasibility of cerebral blood volume mapping by flat panel detector CT in the angiography suite: first experience in patients with acute middle cerebral artery occlusions. AJNR Am J Neuroradiol 2012;33:618-25

12. Struffert T, Deuerling-Zheng Y, Kloska S, et al. Cerebral blood volume imaging by flat detector computed tomography in comparison to conventional multislice perfusion CT. Eur Radiol 2011;21:882-89
13. Struffert T, Deuerling-Zheng Y, Kloska S, et al. Flat detector CT in the evaluation of brain parenchyma, intracranial vasculature, and cerebral blood volume: a pilot study in patients with acute symptoms of cerebral ischemia. AJNR Am J Neuroradiol 2010;31:1462-69

14. Dörfler A, Struffert T, Engelhorn T, et al. Rotational flat-panel computed tomography in diagnostic and interventional neuroradiology. Rofo 2008;180:891-98

15. Orth RC, Wallace MJ, Kuo MD, et al. C-arm cone-beam CT: general principles and technical considerations for use in interventional radiology. J Vasc Interv Radiol 2008;19:814-20

16. Royalty K, Manhart M, Pulfer K, et al. C-arm CT measurement of cerebral blood volume and cerebral blood flow using a novel highspeed acquisition and a single intravenous contrast injection. AJNR Am J Neuroradiol 2013 May 23. [Epub ahead of print]

17. Amis ES Jr, Butler PF, Applegate KE, et al. American College of Radiology white paper on radiation dose in medicine. J Am Coll Radiol 2007;4:272-84

18. Wintermark M, Lev MH. FDA investigates the safety of brain perfusion CT. AJNR Am J Neuroradiol 2010;31:2-3

19. Staniszewska M. Evaluation of patient exposure in computerised tomography in Poland. Radiat Prot Dosimetry 2002;98:437-40

20. International Commission on Radiological Protection. The $2007 \mathrm{rec}-$ ommendations of the International Commission on Radiological Protection. ICRP Publication 103. Ann ICRP 37 (2-4). http:// www.icrp.org/publication.asp?id=ICRP\%20Publication\%20103. Accessed October 11, 2013

21. Adult Brain Perfusion CT Protocols. Version 1.1. May 22, 2012. http:// www.aapm.org/pubs/CTProtocols/documents/AdultBrainPerfusionCT. pdf. Accessed October 11, 2013

22. Siebert E, Bohner G, Dewey M, et al. 320-slice CT neuroimaging: initial clinical experience and image quality evaluation. Br J Radiol 2009;82:561-70

23. Abels B, Klotz E, Tomandl BF, et al. CT perfusion in acute ischemic stroke: a comparison of 2-second and 1-second temporal resolution. AJNR Am J Neuroradiol 2011;32:1632-39

24. Smith AB, Dillon WP, Gould R, et al. Radiation dose-reduction strategies for neuroradiology CT protocols. AJNR Am J Neuroradiol 2007;28:1628-32

25. Hirata M, Murase K, Sugawara Y, et al. A method for reducing radiation dose in cerebral CT perfusion study with variable scan schedule. Radiat Med 2005;23:162-69

26. Furtado AD, Lau BC, Vittinghoff E, et al. Optimal brain perfusion CT coverage in patients with acute middle cerebral artery stroke. AJNR Am J Neuroradiol 2010;31:691-95

27. Lee DH, Jo KD, Kim HG, et al. Local intraarterial urokinase thrombolysis of acute ischemic stroke with or without intravenous abciximab: a pilot study. J Vasc Interv Radiol 2002;13:769-74

28. Cohnen M, Wittsack HJ, Assadi S, et al. Radiation exposure of patients in comprehensive computed tomography of the head in acute stroke. AJNR Am J Neuroradiol 2006;27:1741-45

29. Olivot JM, Mlynash M, Thijs VN, et al. Relationships between infarct growth, clinical outcome, and early recanalization in diffusion and perfusion imaging for understanding stroke evolution (DEFUSE). Stroke 2008;39:2257-63

30. Arnold M, Schroth G, Nedeltchev K, et al. Intra-arterial thrombolysis in $\mathbf{1 0 0}$ patients with acute stroke due to middle cerebral artery occlusion. Stroke 2002;33:1828-33

31. Söderman M, Babic D, Holmin S, et al. Brain imaging with a flat detector C-arm: technique and clinical interest of XperCT. Neuroradiology 2008;50:863-68

32. Mishra NK, Albers GW, Davis SM, et al. Mismatch-based delayed thrombolysis: a meta-analysis. Stroke 2010;41:e25-33

33. Lin $\mathrm{CJ}, \mathrm{Wu} \mathrm{TH}$, $\mathrm{Lin} \mathrm{CH}$, et al. Can iterative reconstruction improve imaging quality for lower radiation CT perfusion? Initial experience. AJNR Am J Neuroradiol 2013;34:1516-21 